Ibn Al-Haitham Jour. for Pure \& Appl. Sci. 33 (2) 2020

Ibn Al Haitham Journal for Pure and Applied Science

Journal homepage: http://jih.uobaghdad.edu.iq/index.php/j/index

\title{
Bayesian Inference for the Parameter and Reliability Function of Basic Gompertz Distribution under Precautionary loss Function
}

\author{
Manahel Kh. Awad \\ Huda A. Rasheed \\ Department of Mathematics, Collage of Science, University of Mustansiriyah. \\ manahel.kh@yahoo.com \\ hudamath@,uomustansiriyah.edu.iq
}

Article history: Received 15 July 2019, Accepted 15 September 2019, Published in April

2020.

Doi: 10.30526/33.2.2435

\begin{abstract}
In this paper, some estimators for the unknown shape parameter and reliability function of Basic Gompertz distribution have been obtained, such as Maximum likelihood estimator and Bayesian estimators under Precautionary loss function using Gamma prior and Jefferys prior. Monte-Carlo simulation is conducted to compare mean squared errors (MSE) for all these estimators for the shape parameter and integrated mean squared error (IMSE's) for comparing the performance of the Reliability estimators. Finally, the discussion is provided to illustrate the results that summarized in tables.
\end{abstract}

Keywords: Basic Gompertz distribution, Maximum likelihood estimator, Bayes estimator, Precautionary loss function, Mean squared errors.

\section{Introduction}

The Gompertz distribution was originally introduced by Benjamin Gompertz in 1825. It is important in describing the pattern of adult deaths and actuarial tables. The Gompertz distribution has many real life applications, especially in medical and actuarial studies and is also used as a survival model in reliability [1].

Reliability theory has played a major role in the rapid interest in the study of models of failure. This theory deals with the study of ages to investigate and study the behavior failure time at posterior time. Reliability theory is one of those standards that show the performances of any unite for an expected period of time. It is also considered an indicator for future planning [2].

The probability density function of the Gompertz distribution is defined as follows [3]:

$\mathrm{f}(\mathrm{t} ; \lambda)=\lambda \exp \left[\mathrm{ct}+\frac{\lambda}{c}\left(1-\mathrm{e}^{\mathrm{ct}}\right)\right] ; \mathrm{t} \geq 0 \quad c, \lambda>0$

where $\mathrm{c}$ is the scale parameter and $\lambda$ is the scale parameter of the Gompertz distribution. 
In this paper, we'll assume that $\mathrm{c}=1$ which is a special case of Gompertz distribution known as Basic Gompertz distribution with the following probability density function:

$\mathrm{f}(\mathrm{t} ; \lambda)=\lambda \exp \left[\mathrm{t}+\lambda\left(1-\mathrm{e}^{\mathrm{t}}\right)\right] ; \mathrm{t} \geq 0, \lambda>0$

The corresponding cumulative distribution function $\mathrm{F}(\mathrm{t})$ is given by [3]:

$\mathrm{F}(\mathrm{t})=1-\exp \left[\lambda\left(1-\mathrm{e}^{\mathrm{t}}\right)\right] \quad ; \mathrm{t} \geq 0$

The reliability or survival function $\mathrm{R}(\mathrm{t})$ and hazard rate $\mathrm{H}(\mathrm{t})$ of Basic Gompertz distribution are defined as

$\mathrm{R}(\mathrm{t})=\overline{\mathrm{F}(\mathrm{t})}=\exp \left[\lambda\left(1-\mathrm{e}^{\mathrm{t}}\right)\right] \quad ; \mathrm{t} \geq 0$

The hazard function $\mathrm{h}(\mathrm{t})$ which is devoted to the rate of failure of the system immediately after time $t$, is defined as:

$\mathrm{h}(\mathrm{t})=\frac{\mathrm{f}(\mathrm{t})}{\mathrm{R}(\mathrm{t})}=\frac{\lambda \exp \left[\mathrm{t}+\lambda\left(1-\mathrm{e}^{\mathrm{t}}\right)\right]}{\exp \left[\lambda\left(1-\mathrm{e}^{\mathrm{t}}\right)\right]}=\lambda \exp (\mathrm{t})$

Respectively,

\section{Maximum Likelihood Estimator of the Shape Parameter $(\lambda)[4,5]$.}

Let $\underline{t}=t_{1}, t_{2}, \ldots, t_{n}$ be a random sample of size $n$ from the Basic Gompertz distribution defined by eq. (1), the likelihood function for the sample observation will be as follows:

$\mathrm{L}\left(\mathrm{t}_{1}, \mathrm{t}_{2}, \ldots, \mathrm{t}_{\mathrm{n}} ; \lambda\right)=\pi_{\mathrm{i}=1}^{\mathrm{n}} \mathrm{f}\left(\mathrm{t}_{\mathrm{i}} ; \lambda\right)=\lambda^{\mathrm{n}} \exp \left[\sum_{\mathrm{i}=1}^{\mathrm{n}} \mathrm{t}_{\mathrm{i}}+\lambda \sum_{\mathrm{i}=1}^{\mathrm{n}}\left(1-\mathrm{e}^{\mathrm{t}_{\mathrm{i}}}\right)\right]$

The natural log-likelihood function will be:

$\ln L\left(t_{i} ; \lambda\right)=n \ln \lambda+\sum_{i=1}^{n} t_{i}+\lambda \sum_{i=1}^{n}\left(1-e^{t_{i}}\right)$

By differentiating partially $\ln L\left(t_{i} ; \lambda\right)$ given above with respect to $\lambda$ and equate to zero, the MLE of $\lambda$ become:

$$
\begin{aligned}
\hat{\lambda}_{\mathrm{ML}} & =\frac{-\mathrm{n}}{\sum_{\mathrm{i}=1}^{\mathrm{n}}\left(1-\mathrm{e}^{\mathrm{t}_{\mathrm{i}}}\right)} \\
& =\frac{-\mathrm{n}}{\mathrm{T}}
\end{aligned}
$$

Where $\mathrm{T}=\sum_{\mathrm{i}=1}^{\mathrm{n}}\left(1-\mathrm{e}^{\mathrm{t}_{\mathrm{i}}}\right)$

\section{Bayesian Estimation}

\subsection{Posterior Density Functions Using Gamma Distribution [6, 7].}

In this section, Gamma distribution had been considered as a prior distribution of the shape parameter $\lambda$ which is defined as follows:

$g(\lambda)=\frac{\beta^{\alpha}}{\Gamma(\alpha)} \lambda^{\alpha-1} \mathrm{e}^{-\beta \lambda} \quad \alpha, \beta>0 \quad ; \lambda>0$

The posterior probability density function of the shape parameter $\lambda$ can be expressed as form

$h_{1}(\lambda \mid \underline{t})=\frac{L\left(t_{1}, t_{2}, \ldots, t_{n} ; \lambda\right) g(\lambda)}{\int_{\forall \lambda} L\left(t_{1}, t_{2}, \ldots, t_{n} ; \lambda\right) g(\lambda) d \lambda}$

Now, combining eq. (3) with eq. (5) in eq. (6), yields: 
$\mathrm{h}_{1}(\lambda \mid \underline{\mathrm{t}})=\frac{\lambda^{\mathrm{n}+\alpha-1} \mathrm{e}^{-\lambda(\beta-T)}}{\int_{0}^{\infty} \lambda^{\mathrm{n}+\alpha-1} \mathrm{e}^{-\lambda(\beta-T)} \mathrm{d} \lambda}$

After simplification, we get:

$\mathrm{h}_{1}(\lambda \mid \underline{\mathrm{t}})=\frac{(\beta-\mathrm{T})^{\mathrm{n}+\alpha} \lambda^{\mathrm{n}+\alpha-1} \mathrm{e}^{-\lambda(\beta-\mathrm{T})}}{\Gamma(\mathrm{n}+\alpha)}$

The posterior pdf of the parameter $\lambda$ is obviously Gamma distribution, i.e.,

$\lambda \mid \underline{t} \sim \operatorname{Gamma}(n+\alpha,(\beta-T))$, with

$\mathrm{E}(\lambda \mid \underline{t})=\frac{\mathrm{n}+\alpha}{\beta-\mathrm{T}} \quad, \operatorname{Var}(\lambda \mid \underline{t} \sim \lambda)=\frac{\mathrm{n}+\alpha}{(\beta-T)^{2}}$

\subsection{Posterior Density Functions Using Jeffreys Prior [8].}

Let us assume that $\lambda$ has non-informative prior density defined as using Jeffreys prior information $g(\lambda)$ which given by:

$g(\lambda) \propto \sqrt{\mathrm{I}(\lambda)}$

Where $I(\lambda)$ represented Fisher information which defined as follows:

$\mathrm{I}(\lambda)=-\mathrm{nE}\left(\frac{\partial^{2} \operatorname{lnf}(t, \lambda)}{\partial \lambda^{2}}\right)$

Hence,

$$
\begin{aligned}
& \mathrm{g}(\lambda)=\mathrm{k} \sqrt{-\mathrm{nE}\left(\frac{\partial^{2} \operatorname{lnf}(\mathrm{t} ; \lambda)}{\partial \lambda^{2}}\right)}, \quad \mathrm{k} \text { is a constant } \\
& \operatorname{lnf}(t, \lambda)=\ln \lambda+\mathrm{t}+\lambda\left(1-\mathrm{e}^{\mathrm{t}}\right) \\
& \frac{\partial \operatorname{lnf}}{\partial \lambda}=\frac{1}{\lambda}+\left(1-\mathrm{e}^{\mathrm{t}}\right) \\
& \frac{\partial^{2} \operatorname{lnf}}{\partial \lambda^{2}}=-\frac{1}{\lambda^{2}}
\end{aligned}
$$

Thus, we get, $\mathrm{E}\left(\frac{\partial^{2} \ln f(t ; \lambda)}{\partial \lambda^{2}}\right)=-\frac{1}{\lambda^{2}}$

After substitution into eq. (7) yields,

$$
g(\lambda)=\frac{\mathrm{k}}{\lambda} \sqrt{\mathrm{n}} \quad, \quad \lambda>0
$$


Then, the posterior density function based on Jeffreys prior is:

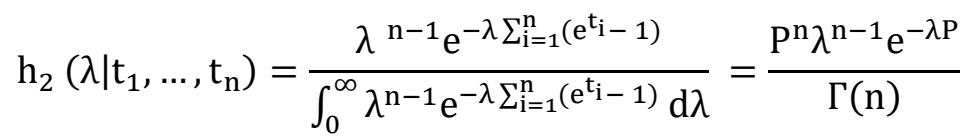

Where $\mathrm{P}=\sum_{\mathrm{i}=1}^{\mathrm{n}}\left(\mathrm{e}^{\mathrm{t}_{\mathrm{i}}}-1\right)$

The posterior density is recognized as the density of the Gamma distribution, i.e.

$\left(\lambda \mid t_{1}, \ldots, t_{n}\right) \sim$ Gamma $(n, P)$, with [9].

\subsection{Bayes Estimation under Precautionary Loss Function [10].}

Precautionary loss function, which is proposed by Norstrom (1996), is one of asymmetric loss function, which can be defined as follows [11].

$$
\mathrm{L}(\hat{\lambda}, \lambda)=\frac{(\lambda-\widehat{\lambda})^{2}}{\widehat{\lambda}}
$$

The Precautionary loss function approach infinitely near the origin to prevent under estimation, thus giving conservative estimators, especially when low failure rates are being estimated. It is very useful when underestimation may lead to serious consequences. For instance, in the case of estimation of a financial charge or size of an order, underestimation has much more serious consequences.

It can be deriving risk function and Bayes estimator for the parameter $\lambda$ in $R_{P B}(\hat{\lambda}, \lambda)$ and $\hat{\lambda}_{P}$ based on Precautionary loss function as follows:

$$
\begin{aligned}
\mathrm{R}_{\mathrm{PB}}(\hat{\lambda}, \lambda) & =\mathrm{E}[\mathrm{L}(\hat{\lambda}, \lambda)] \\
\mathrm{R}_{\mathrm{PB}}(\hat{\lambda}, \lambda) & =\int_{0}^{\infty} \mathrm{L}(\hat{\lambda}, \lambda) \mathrm{h}(\lambda \mid \underline{\mathrm{x}}) \mathrm{d} \lambda \\
\mathrm{R}_{\mathrm{PB}}(\hat{\lambda}, \lambda) & =\int_{0}^{\infty} \frac{(\lambda-\hat{\lambda})^{2}}{\hat{\lambda}} \mathrm{h}(\lambda \mid \underline{\underline{x}}) \mathrm{d} \lambda \\
& =\int_{0}^{\infty}\left(\lambda^{2} \hat{\lambda}^{-1}\right) \mathrm{h}(\lambda \mid \underline{\underline{x}}) \mathrm{d} \lambda-\int_{0}^{\infty} 2 \lambda \mathrm{h}(\lambda \mid \underline{x}) \mathrm{d} \lambda+\int_{0}^{\infty} \hat{\lambda} \mathrm{h}(\lambda \mid \underline{x}) \mathrm{d} \lambda \\
\mathrm{R}_{\mathrm{PB}}(\hat{\lambda}, \lambda) & =\mathrm{E}\left(\lambda^{2} \mid \underline{x}\right) \hat{\lambda}^{-1}-2 \mathrm{E}(\lambda \mid \underline{x})+\hat{\lambda}
\end{aligned}
$$

Taking the partial derivative for $\mathrm{R}_{\mathrm{PB}}(\hat{\lambda}, \lambda)$ with respect to $\hat{\lambda}$ and setting it equal to zero, gives:

$$
\begin{aligned}
\hat{\lambda}^{2} & =E\left(\lambda^{2} \mid \underline{x}\right)=\int_{0}^{\infty} \lambda^{2} h(\lambda \mid \underline{x}) d \lambda \\
& =\sqrt{E\left(\lambda^{2} \mid \underline{x}\right)}
\end{aligned}
$$




\subsubsection{Bayes estimation under Precautionary Loss Function with Gamma Prior}

Bayes estimator relative to Precautionary loss function based on Gamma prior, can be derived as follows:

$$
\begin{aligned}
& \mathrm{E}\left(\lambda^{2} \mid \underline{\mathrm{x}}\right)=\int_{0}^{\infty} \lambda^{2} \frac{(\beta-\mathrm{T})^{\mathrm{n}+\alpha}}{\Gamma(\mathrm{n}+\alpha)} \lambda^{\mathrm{n}+\alpha-1} \mathrm{e}^{-\lambda(\beta-\mathrm{T})} \mathrm{d} \lambda \\
& \quad=\frac{(\beta-\mathrm{T})^{\mathrm{n}+\alpha} \Gamma(\mathrm{n}+\alpha+2)}{\Gamma(\mathrm{n}+\alpha)(\beta-\mathrm{T})^{\mathrm{n}+\alpha+2}} \int_{0}^{\infty} \frac{(\beta-\mathrm{T})^{\mathrm{n}+\alpha+2}}{\Gamma(\mathrm{n}+\alpha+2)} \lambda^{\mathrm{n}+\alpha+1} \mathrm{e}^{-\lambda(\beta-T)} \mathrm{d} \lambda \\
& \mathrm{E}\left(\lambda^{2} \mid \underline{\mathrm{x}}\right)=\frac{(\mathrm{n}+\alpha)(\mathrm{n}+\alpha+1)}{(\beta-\mathrm{T})^{2}}
\end{aligned}
$$

Thus, Bayesian estimation for the shape parameter of Basic Gompartz distribution under precautionary loss function with Gamma prior, denoted by $\hat{\lambda}_{\mathrm{PBG}}$ is

$$
\hat{\lambda}_{\mathrm{PBG}}=\sqrt{\frac{(\alpha+\mathrm{n})(\alpha+\mathrm{n}+1)}{(\beta-\mathrm{T})^{2}}}
$$

Now, Bayesian estimation for Reliability function under Precautionary loss function can be obtained using eq. (8), as follows

$$
\begin{aligned}
& \widehat{R}(t)=\sqrt{E\left((R(t))^{2} \mid \underline{t}\right)} \\
& \left.E(R(t))^{2} \mid \underline{t}\right)=\int_{0}^{\infty}\left(e^{\lambda\left(1-e^{t}\right)}\right)^{2} \frac{(\beta-T)^{n+\alpha}}{\Gamma(n+\alpha)} \lambda^{n+\alpha-1} e^{-\lambda(\beta-T)} d \lambda \\
& =\left(\frac{(\beta-T)}{\left((\beta-T)-2\left(1-e^{t}\right)\right.}\right)^{n+\alpha} \int_{0}^{\infty} \frac{\left((\beta-T)-2\left(1-e^{t}\right)^{n+\alpha}\right.}{\Gamma(n+\alpha)} \lambda^{n+\alpha-1} e^{-\lambda\left((\beta-T)-2\left(1-e^{t}\right)\right)} d \lambda \\
& \left.E(R(t))^{2} \mid \underline{t}\right)=\left(\frac{(\beta-T)}{\left((\beta-T)-2\left(1-e^{t}\right)\right.}\right)^{n+\alpha}
\end{aligned}
$$

Combining equations (10) and (11) gives

$$
\widehat{\mathrm{R}}(\mathrm{t})_{\mathrm{PBG}}=\sqrt{\left(\frac{(\beta-\mathrm{T})}{\left((\beta-\mathrm{T})-2\left(1-\mathrm{e}^{\mathrm{t}}\right)\right.}\right)^{\mathrm{n}+\alpha}}
$$

\subsubsection{Bayes Estimation under Precautionary Loss Function with Jefferys Prior}

The Bayes estimator for the shape parameter $\lambda$ under Jefferys prior can be obtained using equation (8) as follows:

$\mathrm{E}\left(\lambda^{2} \mid \underline{\mathrm{x}}\right)=\int_{0}^{\infty} \lambda^{2} \frac{\mathrm{P}^{\mathrm{n}}}{\Gamma(\mathrm{n})} \lambda^{\mathrm{n}-1} \mathrm{e}^{-\lambda \mathrm{P}} \mathrm{d} \lambda=\frac{\mathrm{P}^{\mathrm{n}} \Gamma(\mathrm{n}+2)}{\Gamma(\mathrm{n}) \mathrm{P}^{\mathrm{n}+2}} \int_{0}^{\infty} \frac{\mathrm{P}^{\mathrm{n}+2}}{\Gamma(\mathrm{n}+2)} \lambda^{\mathrm{n}+1} \mathrm{e}^{-\lambda \mathrm{p}} \mathrm{d} \lambda$

Therefore, $\quad E\left(\lambda^{2} \mid \underline{x}\right)=\frac{n(n+1)}{P^{2}}$ 
$\hat{\lambda}_{\mathrm{PBJ}}=\sqrt{\frac{\mathrm{n}(\mathrm{n}+1)}{\mathrm{p}^{2}}}$

Similarly, we can find the estimator for Reliability function under Precautionary loss function, by using eq. (8), where:

$$
\begin{aligned}
& \widehat{R}(\lambda)=\sqrt{E\left(R^{2} \mid t\right)} \\
& E\left(R^{2}(t) \mid \underline{t}\right)=\int_{0}^{\infty} R^{2} h(\lambda \mid \underline{t}) d \lambda
\end{aligned}
$$

Substituting R(t) into eq. (12), gives:

$$
\begin{aligned}
& \left.E(R(t))^{2} \mid \underline{t}\right)=\int_{0}^{\infty}\left(e^{\lambda\left(1-e^{t}\right)}\right)^{2} \frac{P^{n}}{\Gamma(n)} \lambda^{n-1} e^{-\lambda P} d \lambda=\int_{0}^{\infty} \frac{P^{n}}{\Gamma(n)} \lambda^{n-1} e^{-\lambda\left(P-2\left(1-e^{t}\right)\right)} d \lambda \\
& \left.E(R(t))^{2} \mid t\right)=\left(\frac{P}{\left(P-2\left(1-e^{t}\right)\right.}\right)^{n}
\end{aligned}
$$

Combining eq. (12) with eq.(13) gives,

$$
\widehat{\mathrm{R}}(\mathrm{t})_{\mathrm{PBJ}}=\sqrt{\left(\frac{\mathrm{P}}{\left(\mathrm{P}-2\left(1-\mathrm{e}^{\mathrm{t}}\right)\right.}\right)^{\mathrm{n}}}
$$

\section{Simulation Study}

In this section, a Monte Carlo simulation has been done to compare the performance of the different estimators of the unknown shape parameter for Basic Gompertz distribution $(\lambda)$. The process (L) have been repeated 5000 times with different sample sizes, $\mathrm{n}=15,50$, and100. The values of shape parameter $\lambda$ are chosen as $\lambda=0.5$ and 3. Bayes estimates have been generated with considering two different values for the parameters of Gamma prior, $\alpha$ and $\beta$ as $\alpha=0.8$ and $3, \beta=0.5$ and 3. All estimators that derived in the previous section are evaluated based on their mean squared errors (MSE's), where,

$$
\begin{array}{ll}
\operatorname{MSE}(\lambda)=\frac{\sum_{\mathrm{i}=0}^{\mathrm{L}}\left(\widehat{\lambda}_{\mathrm{i}}-\lambda\right)^{2}}{\mathrm{~L}} ; & \mathrm{i}=1,2,3, \ldots . \mathrm{L} \\
\operatorname{MSE}(\lambda)=\frac{\sum_{\mathrm{i}=0}^{\mathrm{L}} \widehat{\lambda}_{\mathrm{i}}{ }^{2}-2 \lambda \sum_{\mathrm{i}=0}^{\mathrm{L}} \widehat{\lambda}_{\mathrm{i}}+\mathrm{n} \lambda^{2}}{\mathrm{~L}} &
\end{array}
$$

Substituting $\hat{\lambda}$ under Precautionary loss function with Gamma prior that defined in eq. (9) into eq. (14), gives:

$\operatorname{MSE}(\lambda)=\frac{\sum_{\mathrm{i}=0}^{\mathrm{L}}\left[\frac{(\alpha+\mathrm{n})(\alpha+\mathrm{n}+1)}{(\beta-\mathrm{T})^{2}}\right]-2 \lambda \sum_{\mathrm{i}=0}^{\mathrm{L}} \sqrt{\frac{(\alpha+\mathrm{n})(\alpha+\mathrm{n}+1)}{(\beta-\mathrm{T})^{2}}}+\mathrm{n} \lambda^{2}}{\mathrm{~L}}$ 
To compare the performance of the Bayesian estimators for $\mathrm{R}(\mathrm{t})$ the integrated mean squared error (IMSE) has been employed because it is more accurate than MSE.

IMSE is defined as distance between the estimate value of the reliability function and actual value of reliability function which is given by

$\operatorname{IMSE}(\hat{\mathrm{R}}(\mathrm{t}))=\frac{1}{\mathrm{~L}} \sum_{\mathrm{i}=1}^{\mathrm{L}}\left[\frac{1}{\mathrm{n}_{\mathrm{t}}} \sum_{\mathrm{j}=1}^{\mathrm{n}_{\mathrm{t}}}\left(\hat{\mathrm{R}}_{\mathrm{i}}\left(\mathrm{t}_{\mathrm{j}}\right)-\mathrm{R}\left(\mathrm{t}_{\mathrm{j}}\right)\right)\right]^{2}$

$\operatorname{IMSE}(\hat{\mathrm{R}}(\mathrm{t}))=\frac{1}{\mathrm{n}_{\mathrm{t}}} \sum_{\mathrm{j}=1}^{\mathrm{n}_{\mathrm{t}}} \operatorname{MSE}\left(\hat{\mathrm{R}}_{\mathrm{i}}\left(\mathrm{t}_{\mathrm{j}}\right)\right)$,

Where $i=1,2, \ldots, L ; \mathrm{n}_{\mathrm{t}}$ the random limits of $\mathrm{t}_{\mathrm{i}}$.

In this paper, we use $\mathrm{t}=0.1,0.2,0.3,0.4,0.5$.

The results were summarized and tabulated in the following tables for each estimator and for all sample sizes.

Table 1. Expected values and MSE's of the different estimates for the Gompertz distribution when $\lambda=0.5$ under Precautionary loss function.

\begin{tabular}{|c|c|c|c|c|c|c|c|}
\hline \multirow{2}{*}{$\mathbf{n}$} & \multirow{2}{*}{$\begin{array}{c}\text { Estimate } \\
\text { Criteria }\end{array}$} & \multirow{2}{*}{ MLE } & \multirow{2}{*}{$\begin{array}{c}\text { Jeffreys } \\
\text { prior }\end{array}$} & & \multicolumn{4}{|c|}{ Gamma prior } \\
\cline { 5 - 8 } & & & $\boldsymbol{\beta}=\mathbf{0 . 5}$ & $\boldsymbol{\beta}=\mathbf{0}$ & $\boldsymbol{\beta}$ & $\boldsymbol{\alpha}=\mathbf{0 . 5}$ & $\boldsymbol{\beta}=\mathbf{3}$ \\
\hline \multirow{2}{*}{$\mathbf{1 5}$} & $\mathbf{E X P}$ & 0.5353497 & 0.5529070 & 0.5705174 & 0.5217681 & 0.6475865 & 0.5922530 \\
\cline { 5 - 8 } & $\mathbf{M S E}$ & 0.0236000 & 0.0266000 & 0.0292176 & 0.0170183 & 0.0530199 & 0.0298270 \\
\hline \multirow{2}{*}{$\mathbf{5 0}$} & $\mathbf{E X P}$ & 0.5102127 & 0.5152898 & 0.5207399 & 0.5075928 & 0.5430734 & 0.5293627 \\
\cline { 2 - 8 } & $\mathbf{M S E}$ & 0.0056800 & 0.0059200 & 0.0061736 & 0.0052306 & 0.0081020 & 0.0064883 \\
\hline \multirow{2}{*}{$\mathbf{1 0 0}$} & $\mathbf{E X P}$ & 0.5053571 & 0.5078771 & 0.5106167 & 0.5041987 & 0.5217073 & 0.5151500 \\
\cline { 2 - 8 } & $\mathbf{M S E}$ & 0.0026500 & 0.0027100 & 0.0027755 & $\mathbf{0 . 0 0 2 5 4 8 0}$ & 0.0032509 & 0.0028710 \\
\hline
\end{tabular}

Table 2. Expected values and MSE's of the different estimates for the Gompertz distribution when $\lambda=3$ under Precautionary loss function.

\begin{tabular}{|c|c|c|c|c|c|c|c|}
\hline \multirow{2}{*}{$\begin{array}{c}\text { Estimate } \\
\text { Criteria }\end{array}$} & \multirow{2}{*}{ MLE } & \multirow{2}{*}{$\begin{array}{c}\text { Jeffreys } \\
\text { prior }\end{array}$} & & \multicolumn{4}{|c|}{ Gamma prior } \\
\cline { 5 - 8 } & & & $\boldsymbol{\beta}=\mathbf{0 . 5}$ & $\boldsymbol{\beta}=\mathbf{0}$ & $\boldsymbol{\beta}=\mathbf{0 . 5}$ & $\boldsymbol{\alpha}=\mathbf{3}$ \\
\hline \multirow{2}{*}{$\mathbf{1 5}$} & $\mathbf{E X P}$ & 3.2120990 & 3.3174430 & 3.1306090 & 2.0881310 & 3.5535200 & 2.3702080 \\
\cline { 5 - 8 } & $\mathbf{M S E}$ & 0.8484274 & 0.9577731 & 0.6126608 & 0.9436139 & 1.0737720 & 0.5410730 \\
\hline \multirow{2}{*}{$\mathbf{5 0}$} & $\mathbf{E X P}$ & 3.0612750 & 3.0917390 & 3.0455510 & 2.6460040 & 3.1761730 & 2.7594880 \\
\cline { 2 - 8 } & $\mathbf{M S E}$ & 0.2044161 & 0.2130897 & 0.1883017 & 0.2303958 & 0.2335805 & 0.1721351 \\
\hline \multirow{2}{*}{$\mathbf{1 0 0}$} & $\mathbf{E X P}$ & 3.0321400 & 3.0472660 & 3.0251940 & 2.8132220 & 3.0908960 & 2.8743160 \\
\cline { 2 - 8 } & $\mathbf{M S E}$ & 0.0954000 & 0.0976000 & 0.0917271 & 0.1028652 & 0.1033541 & $\mathbf{0 . 0 8 6 7 5 8 7}$ \\
\hline
\end{tabular}


Ibn Al-Haitham Jour. for Pure \& Appl. Sci. 33 (2) 2020

Table 3. IMSE's of the different estimates for $\mathrm{R}(\mathrm{t})$ of Gompertz distribution where $\lambda=0.5$ under Precautionary loss function.

\begin{tabular}{|c|c|c|c|c|c|c|}
\hline \multirow{2}{*}{$\mathbf{n}$} & \multirow{2}{*}{ MLE } & \multirow{2}{*}{$\begin{array}{c}\text { Jeffreys } \\
\text { prior }\end{array}$} & & \multicolumn{4}{|c|}{$\boldsymbol{\alpha}=\mathbf{0 . 8}$} & \multicolumn{2}{|c|}{$\boldsymbol{\alpha}=\mathbf{3}$} \\
\cline { 4 - 7 } & & & $\boldsymbol{\beta}=\mathbf{0 . 5}$ & $\boldsymbol{\beta}=\mathbf{3}$ & $\boldsymbol{\beta}=\mathbf{0 . 5}$ & $\boldsymbol{\beta}=\mathbf{3}$ \\
\hline $\mathbf{1 5}$ & 0.0014595 & 0.0013583 & 0.0011921 & 0.0011893 & 0.0020073 & 0.0007856 \\
\hline $\mathbf{5 0}$ & 0.0003847 & 0.0003768 & 0.0003637 & 0.0003559 & 0.0004424 & 0.0003110 \\
\hline $\mathbf{1 0 0}$ & 0.0001869 & 0.0001850 & 0.0001819 & 0.0001793 & 0.0002019 & $\mathbf{0 . 0 0 0 1 6 7 7}$ \\
\hline
\end{tabular}

Table 4. IMSE's of the different estimates for $\mathrm{R}(\mathrm{t})$ of Gompertz distribution where $\lambda=3$ under Precautionary loss function.

\begin{tabular}{|c|c|c|c|c|c|c|}
\hline \multirow{2}{*}{$\mathbf{n}$} & \multirow{2}{*}{ MLE } & \multirow{2}{*}{$\begin{array}{c}\text { Jeffreys } \\
\text { prior }\end{array}$} & \multicolumn{4}{|c|}{ Gamma prior } \\
\cline { 4 - 7 } & & & $\boldsymbol{\beta}=\mathbf{0 . 5}$ & $\boldsymbol{\beta}=\mathbf{3}$ & $\boldsymbol{\beta}=\mathbf{0 . 5}$ & $\boldsymbol{\beta}=\mathbf{3}$ \\
\hline $\mathbf{1 5}$ & 0.0069471 & 0.0063600 & 0.0099006 & 0.0830026 & 0.0060451 & 0.0661069 \\
\hline $\mathbf{5 0}$ & 0.0021114 & 0.0020500 & 0.0024518 & 0.0186528 & 0.0020164 & 0.0154806 \\
\hline $\mathbf{1 0 0}$ & 0.0010669 & 0.0010500 & 0.0011561 & 0.0062854 & $\mathbf{0 . 0 0 1 0 4 1 5}$ & 0.0053507 \\
\hline
\end{tabular}

\section{Conclusions}

The discussion of the results obtained from applying the simulation study can be summarized as follows:

1. The performance of Bayes estimator under Precautionary loss function of Gompertz distribution is the best estimator comparing to the other estimators (with all sample sizes) when:

- The parameter $\lambda=0.5$ with $\alpha=0.8$ and $\beta=3$ see Table 1 .

- The parameter $\lambda=3$ with $\alpha=3$ and $\beta=3$ see Table 2 .

2. The performance of Bayes estimator under Precautionary loss function for estimating the reliability function $\mathrm{R}(\mathrm{t})$ of Basic Gompertz distribution is the best estimator comparing to the other estimators when:

- The parameter $\lambda=0.5$ with $\alpha=3$ and $\beta=3$ for all sample size see Table 3.

- The parameter $\lambda=3$ with $\alpha=3$ and $\beta=0.5$ for all sample size see Table 4.

\section{References}

1. Wetterstrand, W. Parametric models for life insurance mortality data: Gompertz's law over time. Transactions of the Society of Actuaries.1981, 33, 159-175.

2. Alwan, S.S. Bayes and Empirical Bayes Estimators for Lomax Distribution, Thesis submitted to the Council of the College of Science at Mustansiriyah university, 2015.

3. Rasheed, H.A.; Hasanain, W.S.; Nadia, J. Comparing some estimators of the parameter of Basic Gompertz distribution, Journal of Iraqi Al-Khwarizmi Society (JIKhS).2018, 2, 178-187.

4. Lenart, A. The moments of the Gompertz distribution and maximum likelihood estimation of its parameters, Scandinavian Actuarial Journal.2014, 3, 255-277.

5. Garg, M.L.; Rao, B.R.; Redmond, C.K. Maximum likelihood estimation of the parameters of the Gompertz survival function, Journal of the Royal Statistical Society C. 1970, 19, 152-159. 
Ibn Al-Haitham Jour. for Pure \& Appl. Sci. 33 (2) 2020

6. Griffin, J. E.; Brown, P.J. Inference with normal-gamma prior distributions in regression problems, Bayesian analysis.2010, 5, 1, 171-188.

7. Jaheen, Z.F. A Bayesian analysis of record statistics from the Gompertz model, Applied Mathematics and Computation.2003, 145, 2-3, 307-320.

8. Al-Kutubi, H.S.; Ibrahim, N.A. Bayes estimator for Exponential distribution with extension of Jeffrey prior information, Malaysian Journal of mathematical Sciences. 2009, 3, 2, 297-313.

9. Walck, C. Hand-Book on Statistical Distributions for Experimentalists, Universitey Stockholms, 2007.

10. Li, J.; Ren, H. Estimation of One Parameter Exponential, International Journal Engineering and Manufacturing.2012, 3, 75-81.

11. Naji, L.F.; Rasheed, H.A. Bayesian Estimation for Two Parameters of Gamma Distribution under Generalized Weighted Loss Function, Iraqi Journal of Science.2019, 32, 1, 1161-1171. 